\title{
L'utilisation de l'eau en Israël
}

\author{
Water utilisation in Israel
}

\author{
PAR A. MAYER

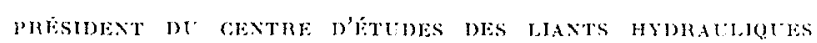

\begin{abstract}
La quantité d'eat lotale susceptible d'ètre recueillie en lsraël ne suffit pas, dans les conditions actuelles, d irriguer les surfaces qui pourraient être rendues disponibles pour l'agriculture. Tout, jusqu'à la derniere gonte, doit etre utilisé dans ce but; il n'est donc pas question de projet hydrólectrique. Celui relatif à l'utilisation de l'eau de la Méditerranée amenée en Mer Morte, malgré l'importante dénivellation $(392 \mathrm{~m})$, n'est pas économique, car il est limité à la quantité d'ean susceptible d'être évaporé par la Mer Morte, sous peine d'en changer le niveau.

Les différents modes d'utilisation de l'eau sont examinés et notamment l'irrigation du Negev a partir des deux fleuves Yarkon et Jourdain.
\end{abstract}

\begin{abstract}
Under present conditions the anount of water that can be collected in Israel is inadequatic for irrigating all the land that conld be made available for agriculture. Every last drop has to be used for this purpose; thas there can be no question of developing hydro-electric power. The plan to use water from the Hedilerranean diverted into the llead Sea, is not economic, in spite of the fact that a $392 \mathrm{~m}$ head is available, because the amounl of water that could be used unould have to be the amount that could be evaporated from the Dead Sea. Otherwise the level of the Dead Sea would rise.

The anthor examines the barious ways of using the water and in particular, discusses the irrigation of the Negen using the waters of the Yarkon and the Jordan.
\end{abstract}

\section{I. - GENERALITES}

Lorsque notre Président me demanda d'exposer à la Société Hydrotechnique de France le problème de l'utilisation de l'eau en Israël, je ne savais pas que cette présentation devait prendre place dans un ensemble relatif aux grands projets hydroélectriques dans le monde. Je lui aurais dit tout de suite qu'il ne pouvait s'agir, en Israël, de projets hydroélectriques, d'abord parce qu'il n'y avait pas, ou pour ainsi dire pas, de sites susceptibles d'être mis en valeur, d'autre part parce que l'eau y était beaucoup trop précieuse pour n'en faire que de l'énergie.

On a pu dire qu'au Moyen-Orient, la richesse naturelle principale était le pétrole; en réalité, ce n'est pas vrai. Seule la présence de l'eau permet de transformer ces pays et de changer la condition de ses habitants. A cet égard, Israël n'est pas un des plus défavorisés, parce qu'au nord de la bande étroite littorale qu'il constitue existe un massif montagneux dans lequel les précipitations annuelles sont de l'ordre du mètre, c'est-à-dire à peu près ce qu'elles sont en
France. Par contre, tout le sud, toute la région du Négev, est aride, avec moins de $10 \mathrm{~cm}$ d'eau par an à l'extrême sud. Or, pour nourrir les deux millions d'habitants qui ont immigré vers Israël au cours des dernic̀res années, ou les quatre millions que l'on considère comme éventuellement susceptibles de s'y trouver à l'avenir, il est indispensable d'utiliser au maximum toutes les régions cultivables, et en particulier de mettre en valeur le Négev. Tout le problème de l'eau en Israël consiste à prendre celle-ci au nord, où elle se trouve relativement en abondance, et à la transporter vers le sud de manière à irriguer le Negev.

Cet aménagement hydraulique a lait l'objet de très nombreuses études d'ordre général. Une des premières a été faite par M. Lowdermilk, qui fut un des pionniers de la Tennessee Valley, et qui, en 1944, rédigea son livre sur la « Palestine, terre promise ». M. Lowdermilk y envisageait la création d'une Jordan Valley Authority à l'instar de ce qui avait été fait dans la 'Ten- 
nessee, et son ouvrage fut accueilli avec grand enthousiasme par la population de la Palestine. Deux groupes d'études furent constitués sous le patronage de l'Agence Juive, l'un à New York, l'autre à Jérusalem, ce dernier présidé par M. Ben Goulion, président actuel du gouvernement d'Israël.

Le bureau technique de New York était présidé par M. Hays, un ingénieur hydraulicien américain. Le résultat des travaux de ces deux Commissions fut la publication, en 1948, d'un rapport intitule "T.V.A. sur le Jourdain », qui parut deux semaines après la création de l'Etat d'Israèl.

L'idée qui avait présidé aux études des deux Commissions était de mettre sur pied un projet susceptible de valoriser non sculement la Palestine, mais encore les zones voisines du Liban et de Jordanie. A cel effet, Ia Commission envisageait non seulement d'utiliser les eaux propres à Israël, mais encore celles dont dispose le Liban et que celui-ci n'utilise pas; en particulier elle prévoyait l'aménagement du cours inícieur du Litani au Liban.

Dès la création de l'Etat d'Israël, le Gouvernement confa au Ministère de l'Agrieulture el à son service des Eaux, dirigé à l'époque par M. Blass, la préparation d'un programme national d'irrigation. Le Service prit alors le nom de Waterplanning for Israël, Ltd. et devint un bureau d'études d'Etat, indépendant, et à burget autonome. La direction technique en fut confice à M. de Leeuw, qui dirigeail à l'époque l'exploilation des Potasses de la Mer Morle. Celui-ci est resté à la tête de cet organisme jusqu'en 1957, date à laquelle il remplaca M. Lowdermilk comme professeur au Technion de Haifa et comme expert international.

Le projet établi par Waterplanning se limite aux frontieres et aux ressources propres de l'Etat d'Israël. Il a fait l'objet de divers examens par un groupe d'experts américains, lesquels, en 1956, en approuvèrent les dispositions.

Avant d'exposer les grandes lignes de ce programme, je reprendrai les diverses caractéristiques de l'Etat d'Israël au point de vue de son approvisionnement en eau.

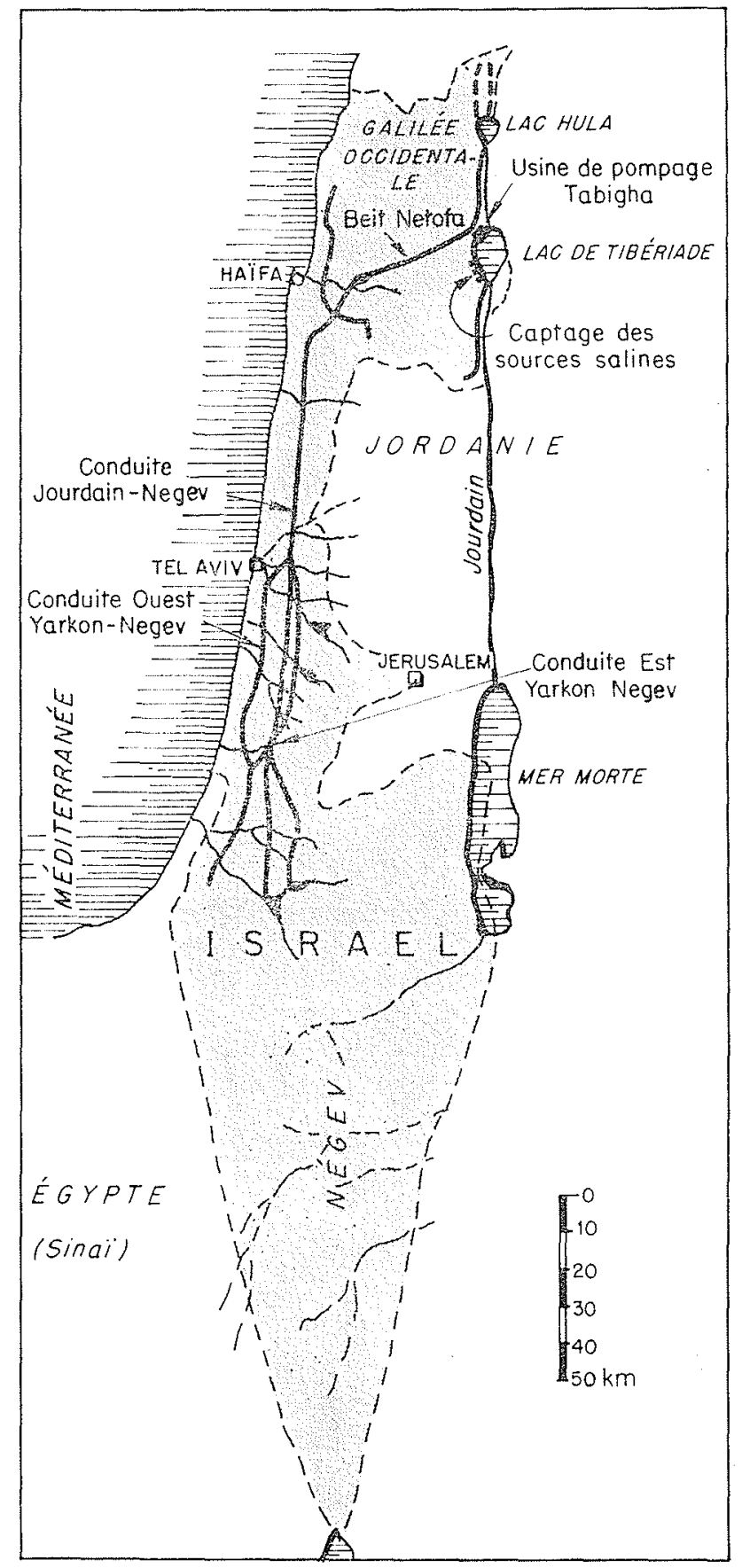

\section{II. - DISPONIBILITÉS}

Comme je l'ai dit, la pluviométrie est variable et passe de $1 \mathrm{~m}$ dans le nord-est du pays a $10 \mathrm{~cm}$ dans le sud du Neger.

Signalons que le pays est recouvert d'une carapace calcaire karstique récente. Les précipi-
Lations à peine tombées s'éraporent ou s'infiltrent. Le taux de ruissellement est extrèmement faible. Toutefois, la présence de couches étanches à certaines profondeurs permet l'aceumulation de nappes importantes, en particulier 
dans la zone côtière. L'utilisation de ces nappes fait bien entendu partie du programme d'ensemble d'aménagement hydraulique de l'Etat d'Israël. Les disponibilités et les différentes sources d'eau sont les suivantes:

D'abord et avant tout le Jourdain. Cette rivière est formée par trois cours d'eau venant tous les trois du Mont Hermon. Deux prennent leux source au Liban, le troisième en Israël même. Le cours du Jourdain, on le sait, est presque exactement nord-sud; descendant du Mont Hermon, il traverse le lac de Tibériade, déjà à $200 \mathrm{~m}$ aurdessous du niveau de la mer, il se jette dans la Mer Morte à la cote -- 392. Le débit moyen est

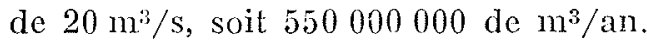

Une autre rivière, le Yarkon, collecte les caux de ruissellement de la zone vallonnée à l'est de Tel Aviv. Elle se jette dans la Méditerrance au nord de cette ville.

Dans notre pays, le Yarkon serait un pelit cours d'eau auquel on n'attacherait aucune importance et le voyageur est surpris, après avoir tant entendu parler des projets de captage des eaux du Yarkon, de constater le faible débit de cette rivière.

Le Jourdain et le Yarkon sont pratiquement les seules rivières d'Israël. Il existe en outre un certain nombre de sources à la limile de la plaine côtière et dans la vallée du Jourdain, qui correspondent à des affleurements des couches imperméables sous la carapace karstique.
On a évalué le débit total des sources et du Yarkion à 425 millions de $\mathrm{m}^{3}$ qui s'écoulent ver's l'ouest et 165 millions de $\mathrm{m}^{3}$ s'écoulant vers l'est, e'est-à-dire vers la vallée du Jourdain.

Les nappes souterraines sont, je l'ai déjà dit, un élément important de l'alimentation en eau d'Israël. Les disponibilités de ces nappes sont mal connues. On a pu les évaluer à 450 millions de $\mathrm{m}^{3} / \mathrm{an}$, une fois l'exploitation en route depuis quelques années. Au début, les quantités disponibles seront très supérieures du fait de l'accumulation de l'eau qui séjourne dans la nappe depuis toujours.

L'inventaire des eaux disponibles en Israël comprend en outre les eaux de crues, dont le volume total a été évalué à 250 millions de $\mathrm{m}^{3} / \mathrm{an}$. On se propose d'en recueillir la moitié dans des retenues convenables.

Restent enfin deux récupérations que l'on compte faire: d'abord 100 millions de $\mathrm{m}^{3}$ sur les eaux d'irrigation, puis la moilié environ des 300 millions de $\mathrm{m}^{3}$ utilisés par la population pour sa consommation propre.

Le total de ces disponibilités est malheureusement insuffisant pour l'irrigation de l'ensemble des terres qui seraient capables de produire. Israël se trouve en présence d'un déficit constant d'eau; il s'agit pour le Gouvernement et le pays d'utiliser l'eau jusqu'à la dernière goutte et de ne pas en laisser partir la moindre fraction à la mer.

\section{III. - ETUDES ET REALISATIONS}

J'ai déjà indiqué la création, en 1952, d'un organisme d'études autonome, bien que relevant directement des pouvoirs publics, dont le nom anglais est Waterplanning for Israël, Ltd. Les premières lettres de la traduction en hébreu de ces mots donnent "Tahal », dénomination sous laquelle ce bureau d'études est généralement connu. Il a la forme d'une société : $52 \%$ des parts sont entre les mains du Gouvernement, $24 \%$ a l'Agence Juive et $24 \%$ an Fonds National Juif. Il s'appuie sur les Services d'Agriculture, Météorologique, Géologique et Géotechnique de l'Etat et sur le Technion d'Haifa et comprend 270 personnes rien qu'en cadres et techniciens. La compétence de ce burcau est actuellement universellement reconnue, si bien que divers pays lui ont confié des études relatives à leur mise en valeur.

Une grande partie des réalisations est confice à une société d'intérêt public, dénommée Meko- roth, fondée en 1938 par l'Agence Juive, le Fonds National Juif et la Centrale syndicale Histadrut. Mekoroth est chargée plus spécialement des sondages et de la construction des canalisations. Cette société a créé deux usines pour la fabrication de tuyaux en acier d'une part, en béton précontraint d'autre part. Elle a diverses filiales pour l'exécution des travaux de sondages et de terrasscment. Son persomnel total est de 5000 ouvriers et cadres.

Les grands travaux à réaliser en Israël sont en général exécutés par l'Entreprise Solel Boneh, entreprise d'Etat, la plus importante probablement du Moyen-Orient et qui, dès à présent, travaille en dehors des limites de l'Etat d'Israël. Elle emploi au total 25000 personnes.

A côté de Solel Boneh, existent d'ailleurs quelques entreprises privées d'importance bien moindre, mais qui permettent aux représentants 
de l'Etat d'Israël de pouvoir affirmer que l'économie n'est pas exclusivement étatiste.

Les moyens d'étude et de réalisation des grands programmes sont done largement suffisants, surtout en raison de leur caractère limité et de la précarité des fonds qui peuvent être utilisés pour les financer. Ces projets n'en présentent pas moins pour le pays une importance vitale. La surface maxima susecptible d'ètre irriguée n'est guère que le quart de la surface totale du pays et les disponibilités en eau, mème évaluées à deux millions de $\mathrm{m}$ \%/an, ne permettent d'irriguer que la moitié de cette surface. Or, dans la plaine côtière, l'irrigation multiplie? les rendements par quatre; au Negev, sans irrigation aucune culture n'est possible; si on a de leau on peut faire deux récoltes par an.

Le programme d'utilisation d'eau en Israël comprend des projets locaux, des projets régionaux et un programme national. Avant d'arriver au programme national, nous dirons quelques mots des projets locaux et régionaux.

\section{Projets locaux.}

Ceux-ci ont, en grande partie, été réalisés par Mekorolh qui a créé, notamment dans la plaine cotière, des stations de pompage par puits ou forages et des installations de captage des sources.

Pendant la période 1952-1956, on a foré $53000 \mathrm{~m}$ de puits ou de forages, mis en place des stations de pompage d'une puissance totale de $125000 \mathrm{ch}$ et on a construit des réservoirs d'une capacité totale de $830000 \mathrm{~m}^{3}$.

Tous ces aménagements comportent des transports d'eau en direction est-ouest, qui pourront un jour être rattachés aux canalisations principales.

\section{Projets régionaux.}

J'en citerai quatre, tous en cours de réalisation par Melioroth.

\section{a) Les marais DE Huleh.}

Les marais de Huleh se trouvent immédiatement au nord du lac de Tibériade, à proximité des sources du Jourdain. C'est un des rares points en Israël où le sol soit constitué par une marne étanche et, lor's de ma première visite en 1950, j’avais suggéré d'y installer le réservoir régulateur nécessaire à l'alimentation de la conduite principale ver's le Negev. Il n'a pas été donné suite à cette suggestion, d'abord parce que des engagements avaient été pris de rendre ce terrain à la culture, d'autre part parce que le réservoir qui aurait conditionné l'existence mêne d'Israël se serait trouvé au contact immédiat de la fronticre jordanienne.

L'assechement des marais de Huleh est pratiquement terminc. On a récupéré 5000 ha pour l'agriculture et la surface tolale irriguce est de 13000 ha. L'cau est amence de la source du Dan par une conduite de $34 \mathrm{~km}$.

\section{b) Irrigation. Région me Beisan.}

Une conduite de $30 \mathrm{~km}$ de $1,80 \mathrm{~m}$ de diamètre capte les sources de la plaine de Beisan et alimente les canaux d'irrigation de celle-ci. Cette canalisation permet également de déverser dans le lac de Tibériade l'excédent des sources en hiver et de récupérer cette eau pendant la saison sèche.

c) Irrigation de la plane de Galilée.

Un programme important est en cours, comprenant l'irrigation de la zone cotière en provenance des sources et des puits dans la plaine au nord de Haïfa. On y déverse également les eaux usées et purifiées de la ville d'Haïfa. Enfin une certaine réserve est constituée dans un bassin en aval d'Haifa pour recueillir les crues de la rivière Kishon.

\section{d) Condutes Yarkon-Negey.}

Il est prévu deux conduites, l'une à l'est, l'autre à l'ouest, pour amener au Negev la plus grande partie des eaux du Yarlion. La moitié environ de ces eaux est captée au roisinage des sources et amenéc vers le sud par une conduite de $1,25 \mathrm{~m}$ en béton. Le reste des eaux sera capté près de la mer. On y ajoutera les eaux de récupération purifiées de l'agglomération de Tel Aviv; une conduite de $1,50 \mathrm{~m}$ est prévue et partiellement construite.

Dès à présent il existe $95 \mathrm{~km}$ de conduite de $1,50 \mathrm{~m}, 12 \mathrm{~km}$ de conduite de $1,25 \mathrm{~m}$ et trois stations de pompage de $18000 \mathrm{ch}$ au total. La capacité totale est de 200000000 de $\mathrm{m}^{3} / \mathrm{an}$, la surface irriguée 37000 ha.

\section{Programme national. La liaison Jourdain-Negev.}

La liaison Jourdain-Negev est le grand programme d'aménagement hydraulique d'Israël, le seul peut-être dont il aurait dù être question au cours de cet exposé.

Comme il a déjà été indiqué, la quantité d'eau disponible est de 550 millons de $\mathrm{m}^{3} /$ an auxquels s'ajoutent 65 millions provenant du drainage des marais de Huleh. On prévoit une utilisation de 300 millions de $\mathrm{m}^{3}$ pouvant ultérieurement être portés à 375 millions de $\mathrm{m}^{3} / \mathrm{an}$ permettant l'irrigation de 55000 ha.

Des trois sources qui alimentent le Jourdain, 
seule celle située en Israël a un débit pratiquement constant, de l'ordre de 10 à $15 \mathrm{~m}^{3} / \mathrm{s}$. Les autres ont un débit variable, qu'il est nécessaire de régulariser. D'autre part, les besoins en eau à l'autre extrómité de la conduite ne sont pas constants. IIs sont évidemment maxima pendant la saison chaude qui est aussi la saison siche. Une régulation annuelle est done indispensable.

Les premiers projets anéricains prévoyaient a cet effet l'utilisation de la plaine de Beit Natufah, laquelle, topographiquement, se prète admirablement à la création d'une retenue.

Lors de ma première visite, en 1950, j'avais attiré l'attention de mes compagnons de royage sur la porosité apparente des roches constituant la retenue et avais fait des réserves sur la possibilite d'utiliser cet emplacement.
Quelques années après, les géologues américains arrivèrent à la même conclusion. Aussi le projet actuel comporte-t-il, comme bassin d'accumulation, le lac de Tibériade lui-même. Il y a là une surface considerable, le fond est bien entendu parfatement étanche, malheureusement il est à $225 \mathrm{~m}$ en contrebas de la canalisation qui l'alimente; d'autre part, il recoit les eaux d'un certain nombre de sources salées gu’il faut détourner si on reut récupérer une grande quantité d'eai pour s'en servir pour les besoins domestiques.

La liaison Jourdain Neger comporte:

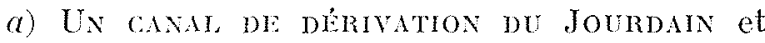
un Bassin D'ACCUMULATION de petite dimension au Wadi Tufah. Ce canal a une longueur de $42 \mathrm{lim}$, il va de la cote 52 a la cote 37 . Sa capa-

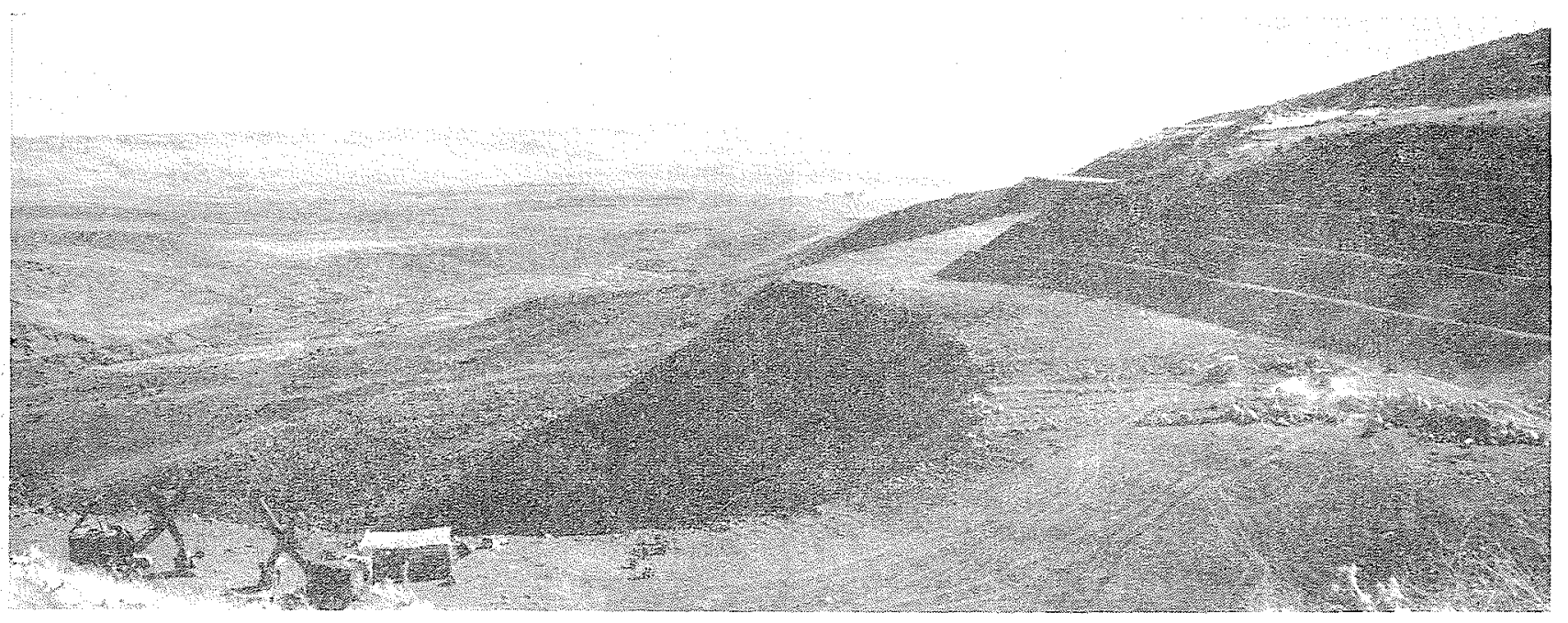

Fic, 1. Travaux préparatoires.

cité est de $25 \mathrm{~m}^{3} / \mathrm{s}$ au départ pour tomber à $20 \mathrm{~m}^{3}$ apres la station de pompage.

Le canal est destiné à transporter par an 435 millions de $\mathrm{m}^{3}$, dont 200 sont envoyés direetement au réservoir du Wadi Tufaì. Les 235 millions restant sont envoyés au lac de Tibériade, qui sert de bassin d'aceumulation.

Pour récupérer les dépenses que le fait d'avoir à pomper cette énorme quantilé d'eau du lac au bassin du Wadi Tufah, c'est-à-dire sur $255 \mathrm{~m}$, a nécessitées, on a equipé la chute on bassin d'acmulation destiné à fournir du courant de pointe au réseau général d'alimenlation du pays, les pompages ćtant faits pendant la nuit sur du courant fourni par le résean a bas prix. La différence de valeur entre le comrant produit et le courant utilisé permet à la fois d'amortir les frais d'installation de la centrale et d'assurer le pompage sur les $120 \mathrm{~m}$ qui correspondent à la différence de nivean entre le Wadi Tufah et le réservoir de Beit Natufah, qui constitue le véritable départ de la conduite.

Comme je l'ai indiqué, ce réservoir devait inilialement servir de bassin inique daccumulation. Actuellement, en raison de l'impossibilité d'étancher l'ensemble du réservoir, on a simplement prévu la constitution, à la sortie de la vallée de Beit Natufah, d'une réserve de faible importance, à un niveau lel que l'cau puisse, de là, s'écouler par simple gravité jusqu'au Negev.

L'usine de Tabigha (prononeer Tabra) comprend une usine géncratrice de $40000 \mathrm{~kW}$ jroduisant du courant de pointe, une station de pompage destince à remonter les eaux du lac vers le réservoir du Wadi Tufah. La capacité de ce réservoir est de 7 millions de $m^{3}$; il peut done 
Fici. …

Irome

des mines.

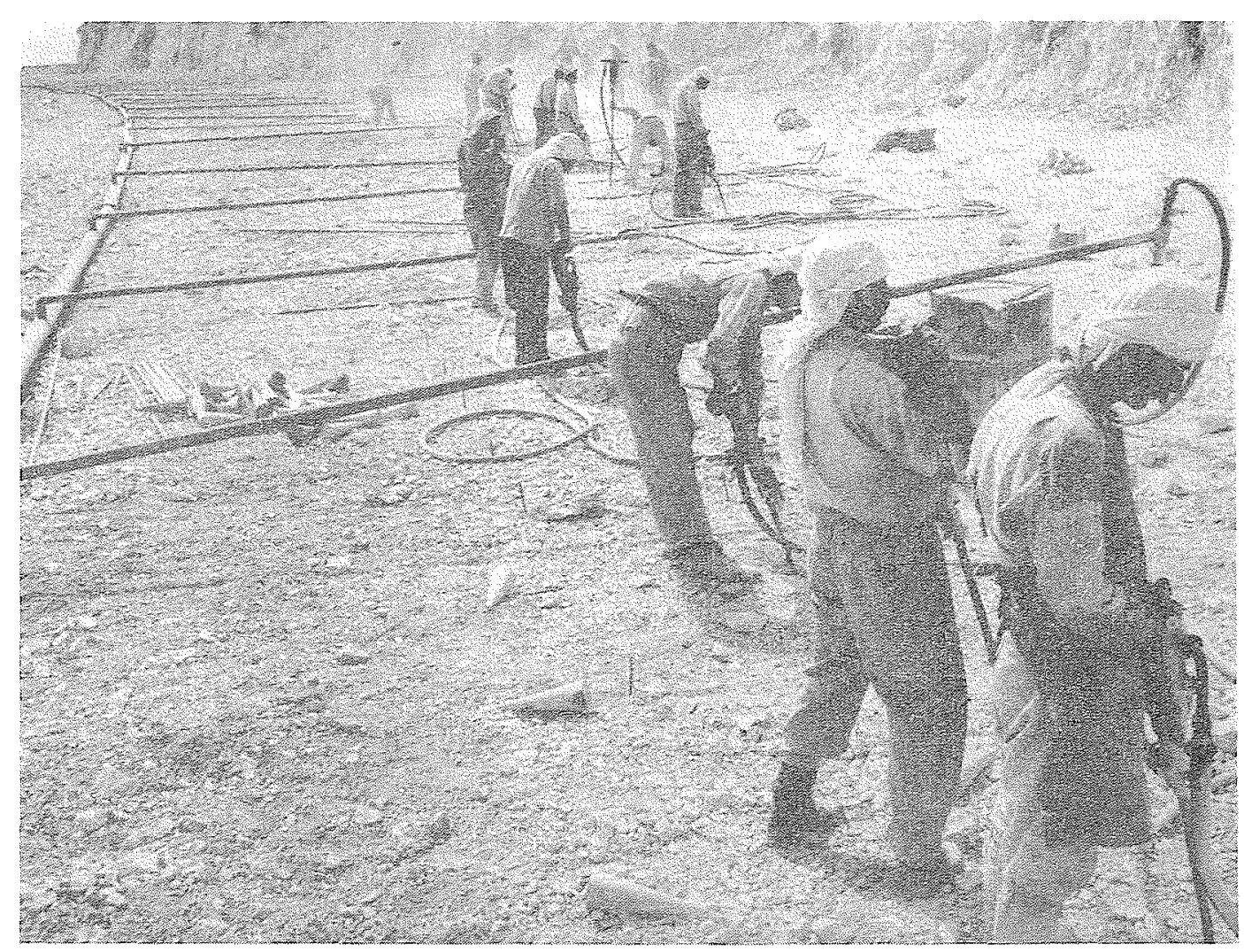

Fig. 3.

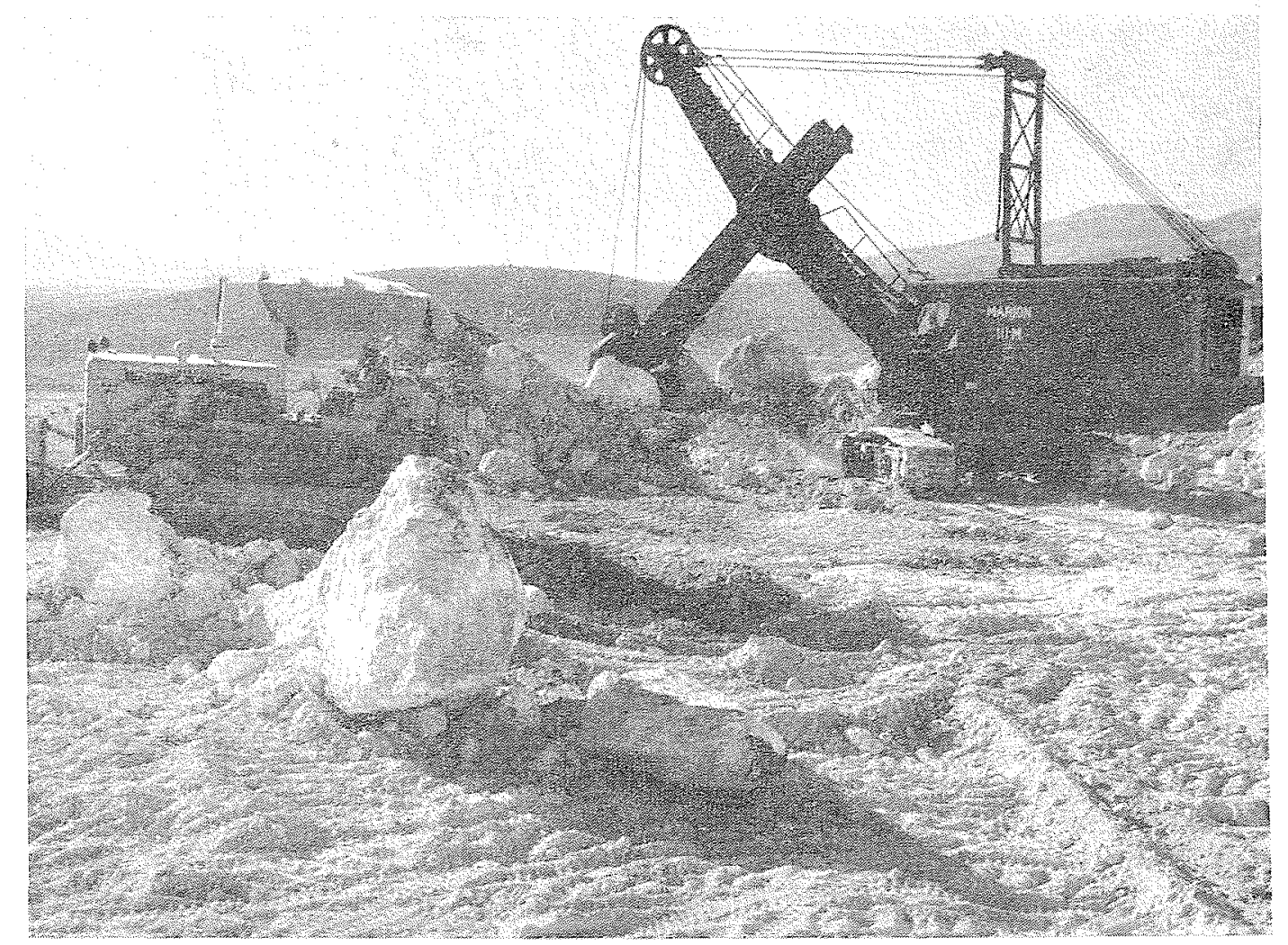




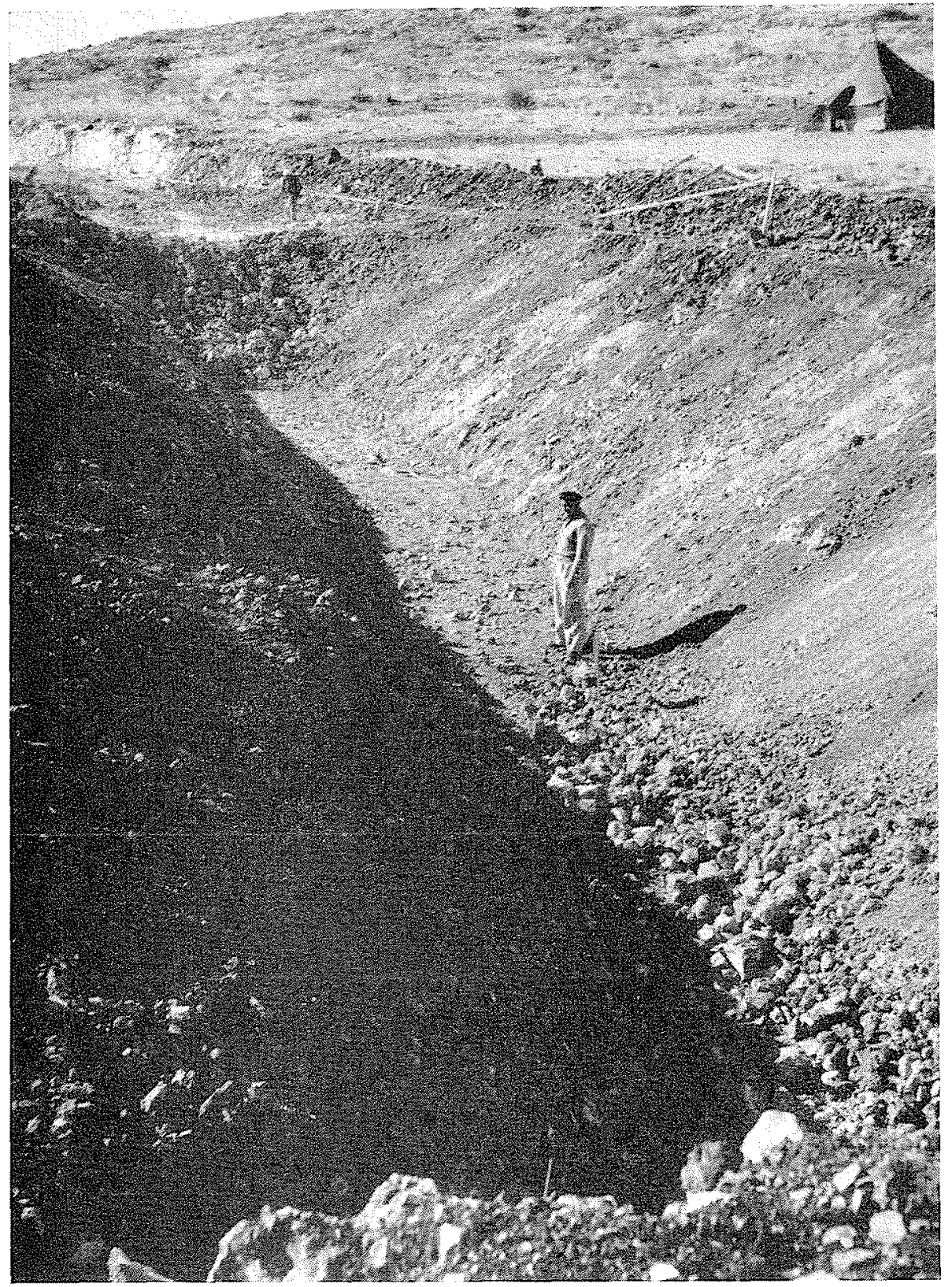

Fili. 4.

Préparation

de la

forme. déjà servir de volant en eas d'immobilisation de l'usine. A signaler qu'entre le Wadi Tufah et Beit Natufah, on a déjà réalisé un tunnel de $850 \mathrm{~m}$ (fig. 6 à 9).

\section{b) Conduite Beit Natufah-Negry.}

Cette conduite est la pièce maitresse de tout le système. En sortant du réservoir de Beit Natufah, welle traverse un tunnel, puis suit la plaine còtière passant de la cote 145 à la cote 85 à l'arrivée à Faluja, où est prévu le centre de distribution. Elle est destinée à transporter 240 millions de $\mathrm{m}^{3}$; la vitesse de circulation sera accélérée par deux stations de pompage.
La conduite est prévue en béton précontraint de $2,50 \mathrm{~m}$ de diamètre. Elle doit constituer le régulateur de l'ensemble du système hydraulique du pays. Tous les réseaux locaux doivent ètre raccordés à cette conduite de manière à avoir une interconnection et à pouvoir parer, le cas échéant, à une insuffisance locale.

Le long de cette conduite, un certain nombre de réservoirs sont prévus, notamment au Wadi Sarrar qui doit pouvoir emmagasiner un volume de 80 millions de $\mathrm{m}^{3}$. Ces réservoirs, bien entendu, servent également à capter les eaux de crues des torrents qui s'y déversent.

Telles sont les grandes lignes de l'aménagement général de l'adduction de l'eau du Jourdain 
au Negev. On a prévu une distribution à partir de Faluja. L'emplacement exact de l'arrivée de la conduite était, à l'époque de ma dernière visite, encore à l'étude, en raison de la difficulté de trouver ou de constituer près de Faluja un réservoir étanche.

\section{IV. - LES PROBLEMES TECHNIQUES}

Après avoir indiqué les grandes lignes de l'aménagement d'Israël, je voudrais dire quelques mots des problemes techniques qui se posent dans ce pays et des conditions dans lesquelles ils ont été étudiés sinon résolus.

\section{a) LUTTE CONTRE L'JUVAPORATION.}

Israël est, à ma connaissance, un des trois seuls pays ou la question de la lutte contre l'évaporation dans les réservoirs situés en pays chaud ait été sérieusement examinée. Des études y ont cté faites s'inspirant des résultats obtenus au Bureau of Reclamation, aux Etats-Unis, et par le Centre de Recherche Scientifique et Industrielle, en Australie. Dans les différents cas, elles ont porté sur des alcools solides à longue chaîne, du type hexadecanol, qur se présentent sous la forme d'un solide cireux plus léger que l'eau. Lorsqu'on dépose un fragment à la surface de l'eau, celui-ci donne une couche monomoléculaire qui se répand progressivement sur toute la surface et constitue une protection semi-étanche contre l'évaporation.

Des essais faits en Israël onl montré que l'étanchéité obtenue n'était pas parfaite. Elle ne devait d'ailleurs pas l'être, à moins de provoquer le développement dans l'eau d'une faune anaérobie, qui rendrait l'eau impropre à la consommation. Néanmoins, les pertes par évaporation sont réduites d'une quantité variant entre le tiers et la moitié, ce qui, dans un pays où l'évaporation atteint et dépasse 1,50 $\mathrm{m}$ par an, a déjà une importante considérable.
Fig. 5.

Bètonnage

de la

forme.

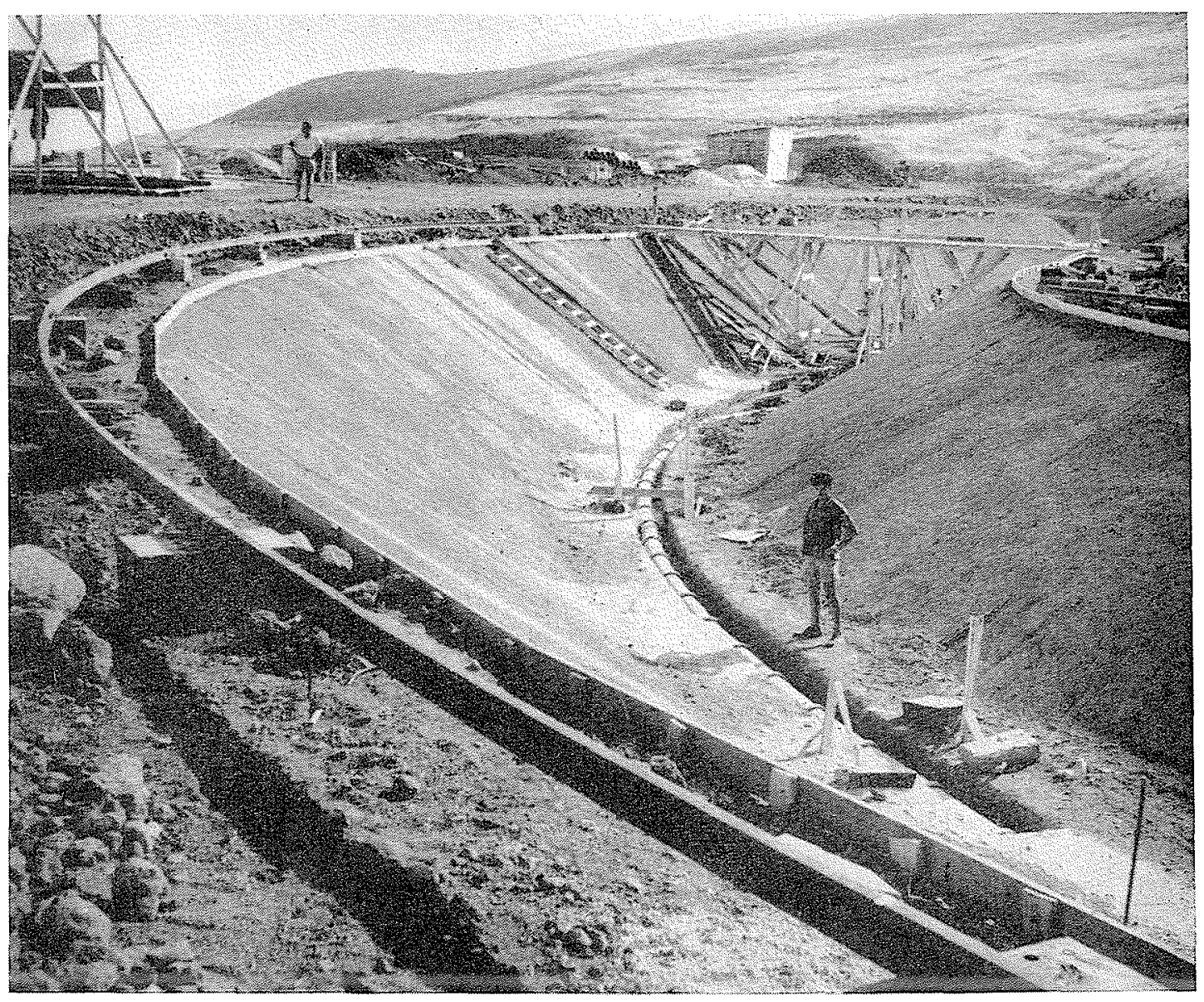




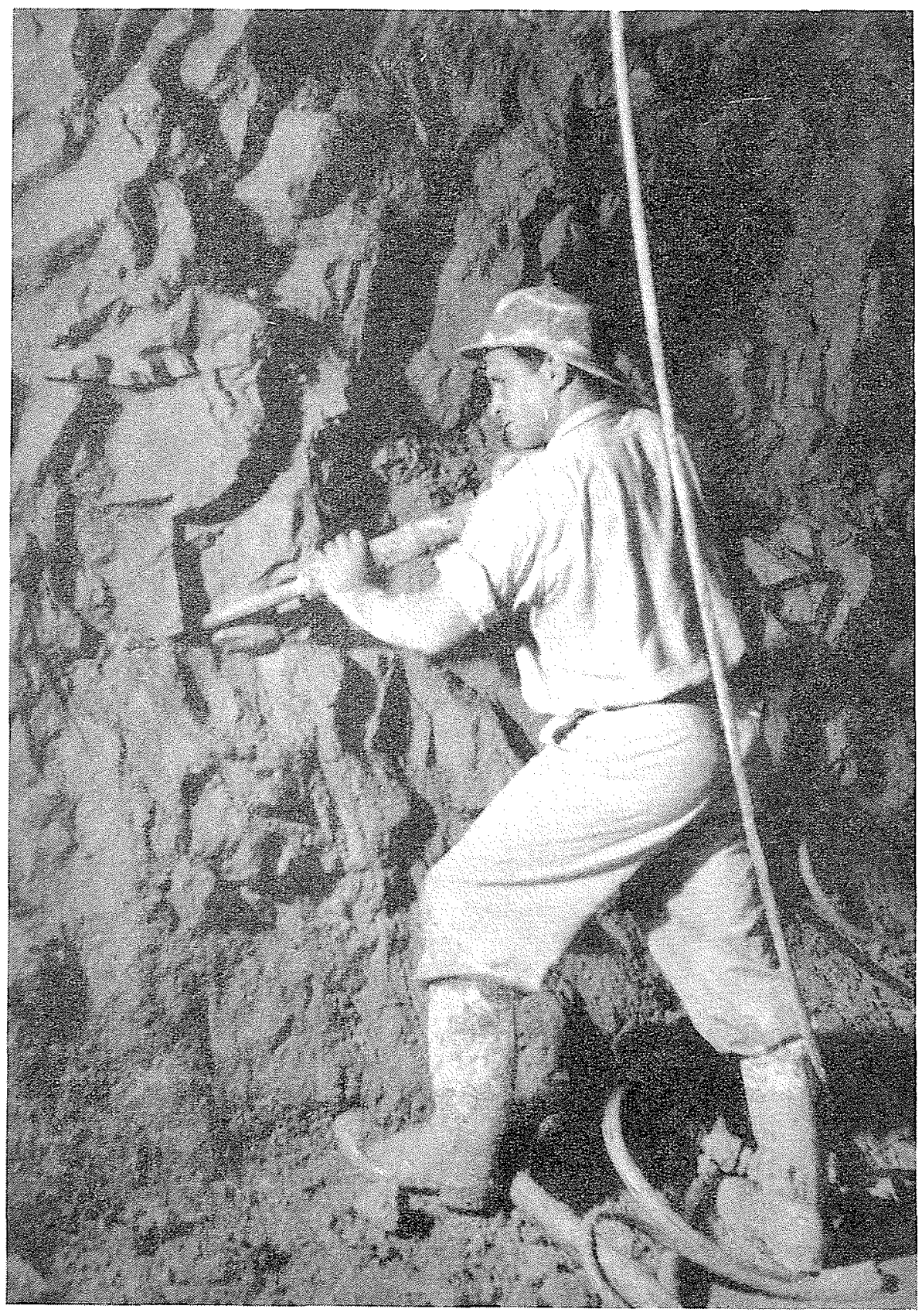

Fis. 6.

Foratge.

Jusqu'ici, à ma connaissance, le procédé n'a pas été appliqué à grande échelle; les essais de laboratoire en ont toutefois montré l'intérêt.

\section{b) Etanchétté pes réshonolrs.}

La constitution d'un réservoir étanche est le problème principal dans un pays où presque partout la roche est fissuree au point d'être totalement impropre à constituer une retenue. L'utilisation de tapis en matériaux limoneux ou argileux doit permettre cependant d'atteindre ce résultat. Il est bien entendu que ceci ne s'appli- que que dans le cas d'une fissuration généralisée de la roche.

Les « ponors \& de Yougoslavie, qui sont des canalisations souterraines atteignant parfois plusieurs mètres de diamètre, nécessitent un traitement tout différent.

La construction de tapis pour la protection do la roche a fait l'objet, en Israël, d'ćtudes systématiques lorsque la question de l'ulilisation de la plaine de Beit Natufah comme réservoir principal s'est posée.

On a constitué deux bassins artificiels de $20 \mathrm{~m} \times 20 \mathrm{~m}$ dans lesquels on a étudié le meil- 


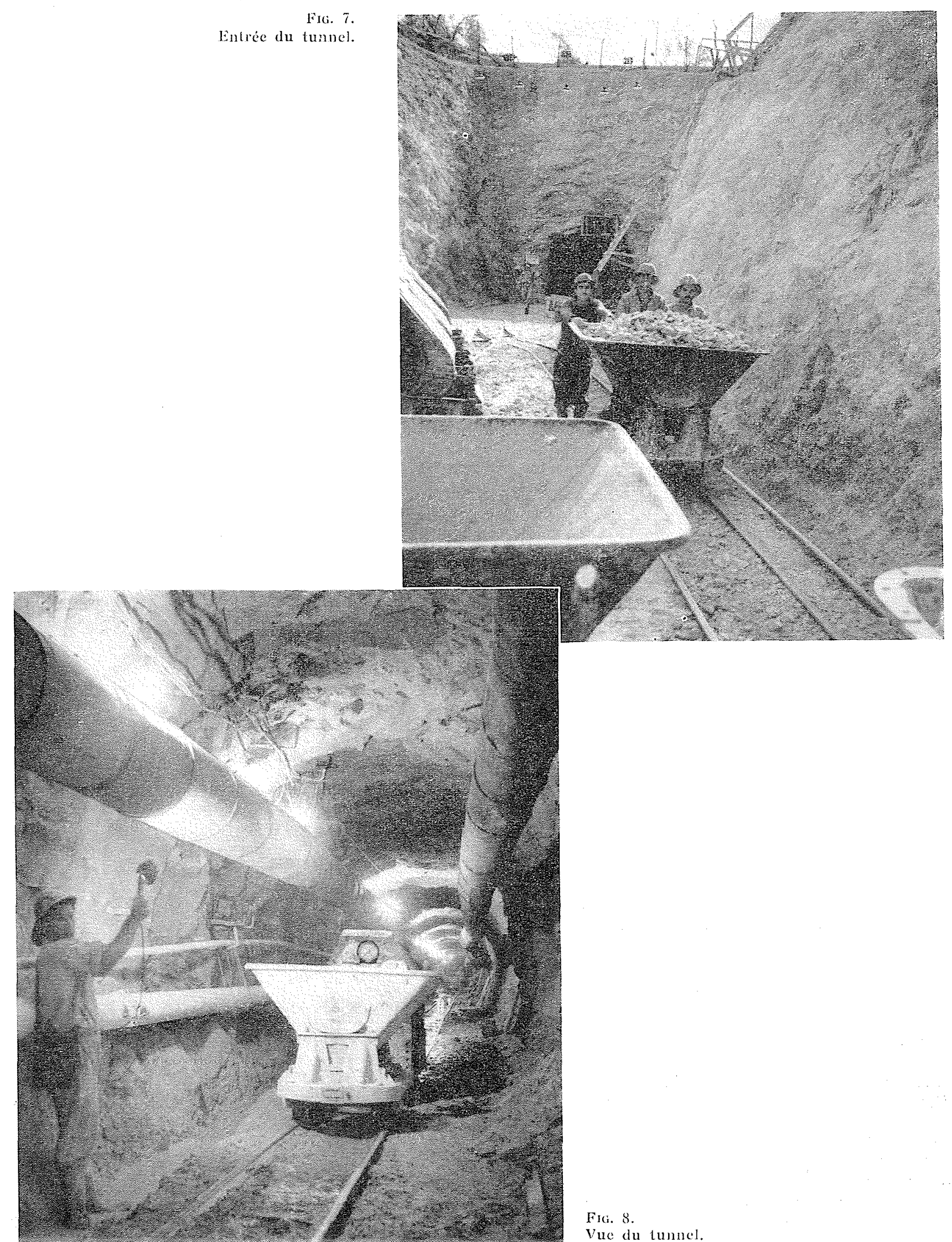




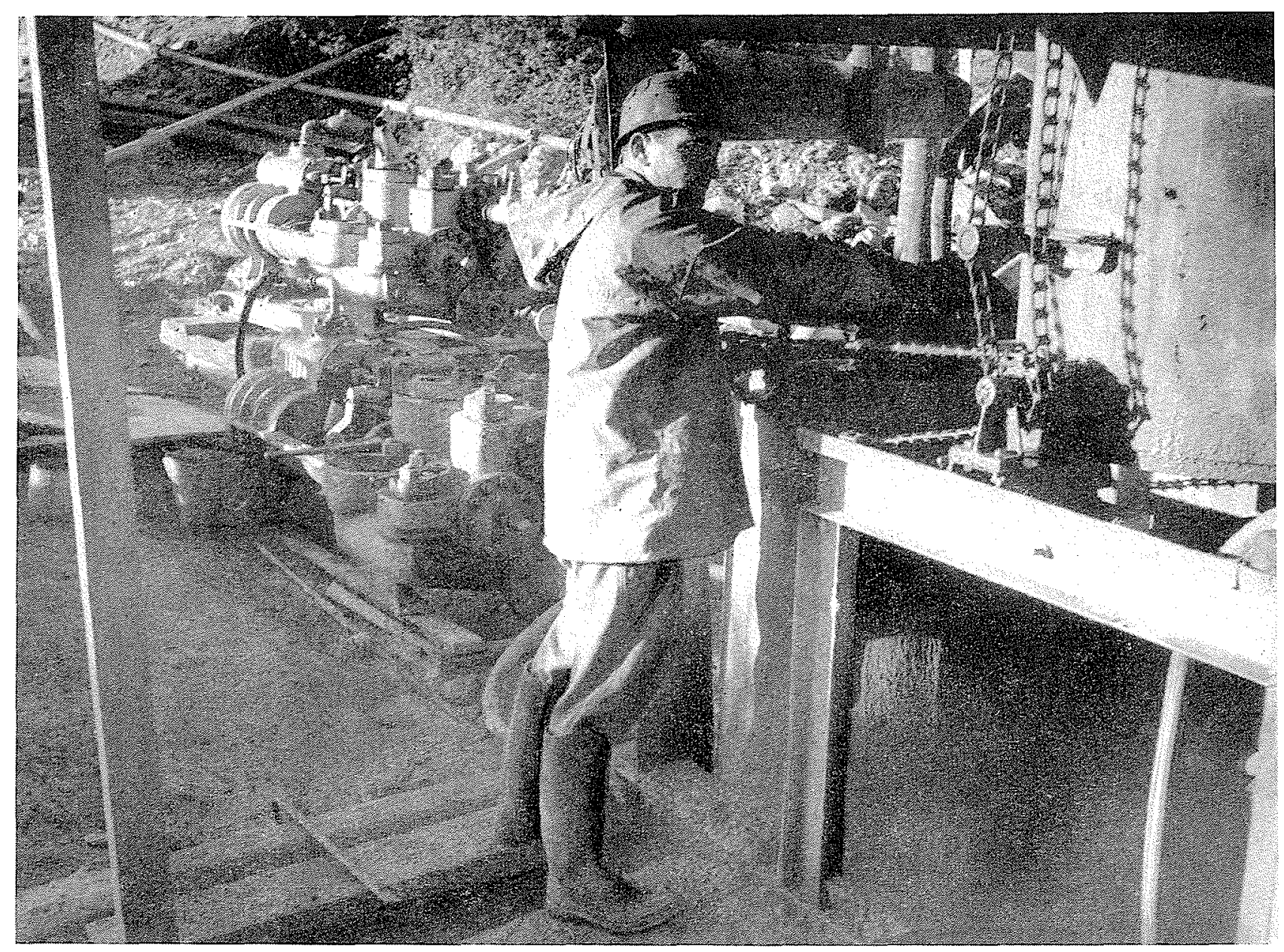

Fig. 9. - Injections d'étanchéité.

leur mode de protection de la roche. Initialement le niveau dans les hassins haissait de plusieurs dizaines de cm par jour. Ia constitution d'un tapis a permis de rédure cette valeur à une quantité comparable aux pertes par évaporation. On a constaté d'ailleurs qu'il ne suffisait pas d'appliquer en surface de la roche un matériau imperméable si le fond des bassins risquait d'être exposé pendant la saison sc̀che à l'action du soleil. Il faut, en partant de la roche, avoir tout d'abord un filtre destiné à retenir les parties fines en cas de fissuration, puis la couche étanche d'au moins $1 \mathrm{~m}$ d'épaisseur constituée par un limon ou une argile corroyée, enfin en surface une protection constituée par une couche d'au moins $25 \mathrm{~cm}$ de cailloux ou d'enrochements destinés à empêcher les animaux d'atteindre la couche étanche.

Une importante série d'essais, d'ailleurs subventionnée par la Fondation Ford, a été faite à Beit Natufah. J'ai eu l'occasion de voir ces essais en cours d'exécution et de suggérer quelques modifications au programme. Une application in- téressante a d'ailleurs été faite de la méthode d'étanchement par tapis dans la retenue de Tel Yeruham, dans le Neger. Celle-ci avait été barrée par un ouvrage en béton assis sur du calcaire fissuré. Le fond de la retenue était constitué par de la marne, mais celle-ci plongeait vers l'aval et était recouverte à l'emplacement du barrage par le calcaire. Il a suffit de rétablir un tapis marneux en surface jusqu'au barrage pour obtenir une étanchéité satisfaisante.

Pratiquement un travail analogue doit être effectué dans la plupart des retenues d'Israël pour assurer leur étanchéité.

\section{c) UTILISATION DU LOESS.}

L'utilisation du loess pour la constitution de digues étanches a également fait l'objet d'études, ce materiau étant, dans certaines parties du Negev, le seul susceptible d'être employé.

L'expérience a prouvé que le loess, après remaniement et mise en place correcte, pouvait constituer des digues d'une stabilité tout à fait satisfaisante, alors qu'en place il comporte des 
vides extrêmement importants dus aux conditions de son dépôt.

\section{d) Construction de tunnels EN MILIEU SATURÉ.}

La construction de certains souterrains a posé des problèmes difficiles du fait de l'existence d'une nappe aquifère dans la roche extrêmement fissurée. Il en est ainsi en particulier de la construction de la cavité destinée à abriter l'usine de pompage de Tabigha, au voisinage du lac de Tibériade.

Les méthodes appliquées en France pour la construction de la galerie d'Issarlès, c'est-à-dire la constitution d'un cylindre étanche par injections, au milieu duquel on a fait la perforation, a permis de faire le creusement avec d'excellents résultats. Il est intéressant à ce propos de noter que les méthodes, mises au point en France et appliquées en Israël, ont été suggérées aux EtatsUnis à une entreprise spécialisée par un ingénieur israélien qui $y$ faisait un stage et $y$ ont permis de résoudre un problème particulic̀rement délicat.

Je n'ai cité ici que les problèmes de ma compétence et que j'ai eu l'occasion d'étudier lors de mes visites en Israël. Il existe au Technion d'Haifa un laboratoire d'hydraulique fort bien monté, dont le directeur est M. Irmaỹ. Celui-ci est de formation belge, ainsi d'ailleurs que M. Aisenstein, le chef de la Section des barrages en terre de Tahal. Ils sont tous deux d'excellents ingénieurs.

\section{V. - LA LIAISON MÉDITERRANEE-MER MORTE}

Il n'a, dans tout cel expose, pas été question. du grand projet qui rient à l'esprit de tout ingénieur qui regarde pour la premiere fois la carte d'Israël : la liaison Méditerranée-Mer Morte.

Une telle liaison permettrait d'utiliser la différence de niveau de prés de $400 \mathrm{~m}$ entre les deux nappes. Le projet est certes séduisant et le serait davantage si la quantité d'ear susceptible d'être prélevée sur la Méditermáe n'était pas limitée par la nécessité de maintenir, approximativement à son niveau actuel, l'eau de la Mer Morte sous peine de noyer les installations industrielles qui se trouvent sur ses bords. On ne peut done faire arriver dans la Mer Morte qu'une quantité d'eau équivalente à celle qui s'évapore. Même si l'on tient compte de la réduction des apports correspondant à la mise en service de la canalisation Jourdain-Negev, l'équipement maximum réalisable ne serait, d'après les études qui m'ont été communiquées, que de $30000 \mathrm{~kW}$ environ.

L'importance des travaux à faire pour amener l'eau de la Méditerranée à la Mer Morte, en raison de la dénivellation qu'il faudrait franchir, rendrait l'opération extrêmement onéreuse. Un calcul sommaire, fait à l'époque de ma dernière visite en Israël, avait montré que le prix de revient $\mathrm{du} \mathrm{kW}$ serait deux fois plus cher que celui d'une centrale thermique installée sur la côte. Il est possible qu'une fois la conduite Jourdain-Negev en fonctionnement, on soit néanmoins amené à réaliser une liaison Méditerranée-Më Morte pour compenser la baisse de niveau de la Mer Morte que produira le détournement des eaux du Jourdain. Pour l'instant, le projet paraît complètement abandonné.

L'utilisation de l'eau en Israël mériterait bien d'autres développements. C'est ainsi que l'irrigation par arrosage en pluie, qui commence seulement à se répandre en France, est là-bas universellement adoptée. L'eau est, dans ce pays, beaucoup trop précieuse pour risquer d'en perdre une fraction importante par infiltation au cours de son transport.

Les techniques les plus modernes sont aussi employées pour la construction des canalisations. On a choisi le béton précontraint de préférence au métal car le ciment est abondant en Israël et fabriqué localement, tandis que le métal doit être importé. Mais l'étude de tous ces facteurs nous entraînerait hor's des limites de cet exposé.

Je tiens, en terminant, à adresser mon meilleur souvenir à tout l'état-major de Tahal ainsi qu'à M. de Leeuw, grâce auquel j'ai fait en Israël ce voyage si intéressant et que j'espère y revoir un jour prochain.

\section{IS C.USSION}

Président : M. Duffaut

M. le Président félicite et remercie M. Mayer de sa très belle conférence et souligne l'intérêt des travaux israéliens présentés.
M. Ie Président remarquant qu'il n'est pas d'usage qu'un pays prenne tout le débit d'un cours d'eau international, M. MaYer confirme que le volume total de l'eau 
du Jourdain, ulilise par Israel ef comprenant la partie dérive el la partie stockie dans le lac de Tiberiande, est de l'ordre de $300000000 \mathrm{~m}^{3} / \mathrm{an}$, alors que le volume total naturel est de l'ordre de $600000000 \mathrm{~m}^{3} / \mathrm{an}$.

M. Je President demande si la possibilite d'ulidiser des reservoirs soulerrains, analogues à cotax gue l'on réalise pour le gat, a ete envisagée pour l'eat coll Isriel ou clle aurat l'avantage d'éviter l'éraporation.

M. Maren répond que ecte possibilite n'a pas ete consideree parce qu'il n'existe pas on qu'il existe tres pea de roches etanches, a part le réservoir naturel roi sin de la cole, constitue par la nappe et exploite par les forases.

M. Chamarov demande si loon a pense à utilisel l'eau de mer en l'adoucissant.

M. Nayen confirme qu'une Section de l'Instilut Scienlifique ssraélien étudie l'adoucissement de l'eau, soit par licraporation qui exige un combustible cher en Isräi, soit par un tratement ehimique gui n'est pas ceonomique non plus.

M. Christian Brat signale que le Gouvernement istaélien lui avail demandé l'année dernière d'examiner, au cours d'une mission sur place, la possibilité de prodiuire de l'énergie et surtout de l'ealu donce, soit à la Mor Horte, soit à la Mer Rouge près d'Eilat, par application des procedés étudies par la Socicté « Finergie des Mers $\gg$.

La question est en suspens en raison des difficultes propres a ces cas tres particuliers qui ne se situent d'ailleurs pas en zone tropicale.

Pour la Mer Morte, il y a bien, tout au moins a certaines heures de la journee, $4 \theta^{\circ} \mathrm{C}$ en surface pendant l'eté, alors que par ailleurs on lroure a ane trentaine ie mitres de profondeur unc couche d'eau froide a $20^{\prime \prime} \mathrm{C}$, ee qui fait apparatre une différence de iemperature de $20^{\circ} \mathrm{C}$. Ces conditions sont extremement intéressantes du point de vue des investissements à consacrer a la conduite d'aspiration d'eau froide. Mais cet avantage est compense par le fait que de telles temperatures de surface n'existent que pendant quelques mois de l'annee. En hiver, la temperature de surface est meme inferieure a celle des profondeurs. En outre, les niveaux de la Mer Morte varient et varieront considerablement en fonction des satisons, de certains cyeles interannuels el enfin des tratrax de derivation du Jourdain actuellement en cours, ce qui complique singulierement la question de l'implantation d'une centrale, étant donné surtout le débit considérable d'eau a prévoir. Par ailleurs, l'extrême salinité de la Mer Morte complique cneore le probleme: à une mome température, la tension de vapent d'une cau très salée est en effet très differente de la fension de la vapeur d'une eau douce ou d'une can de mej normale; en outre, la salinite en Mel Horle augmente considerablement avec la profondeur, car on $y$ constate une densité d'environ 1,16 en surface 1,22 i 30 mètres de protondent et 1,30 vers le fond, ce qui entrane des variations de tensions de vipeur en fonction de ia profondeur, se combinant avec celles provenant des variations de temperatures. Ajoutons enfin que la couche d'eau froide n'est pas considérable et qu'on ne sait pas exactement comment elle se renouvellera.

Du coté de la Mer Rouge prés deilat, on se henrte atu fait qu'en surface, il n'y a guère plus de $27^{\circ} \mathrm{C}$ dans la saison chaude et qu'en profondeur l'eau ne descend pas en dessous de $20^{\circ} \mathrm{C}$ par suite de l'existence de semils trop eleves aux entresen en Mer liouge ef dans le golle d'Akaba, seuils qui empechent l'acees jusqu’a bilat des caux froides oceanes qui, plus denses, sont par conséquent plus protondes. L'ecart de $20^{\circ} \mathrm{C}$ néessaire poul: ane centrale d'eneroic themigue des mers ne pourrait done $y$ etre oblenu que grate a des bassins de rechaufase solatre, lesquels permettrient sans doute d'oblenir comme en Mer Morte des temperatures de l'ordre de $40^{\prime}$ C. D'autres procedes sont an cours d'élude pour obtenis le mene resultal, tels que l'emploi de réseaux de canalisation soutermins a quelques decimetres du sol, l'expérience arant montré que leau douce gui sortait des condules d'adduction situes à une telle profondeur atteignail sourent $10^{\circ} \mathrm{C}$.

En résume, des diffeultés parliculieres existent pour la réalisation en Ismäl de centrates d'energie fhermique les mers susceptibles de produire de l'energie et de I'ealu douce, mais ces difficultés ne paraissent pas insurmontables. Oi l'eau douce, qui peul ainsi efre produite, et d'une facon massive, en même temps que l'electricilé, ne coûtemit pas cher si de telles centrales pouvaient trouver leur éfuilibre économique avec la vente d'énerwie; car le supplément d'investiscment a prévoir pou obtenir de l'eau donce est le remplacement des condenseurs en pluie par des condenseurs à surface qui coûlent un peu plus cher. Il est done vraisemblable que si l'on arrive a une réalisation de ee type, l'ean douce ansj produlte en partant de leau de mer sera plus ceonomique que celle produite en appliquant d'autres yrincipes.

M. René Colas pense qu'on peut etre dtonne par te fait que le procede do dessalage de l'eau de mer par - Gectrodialyse n'ait pas eté poussé davantage en Israël etant donné que linvention en a dé faite par le docteur Walter Juda, il v a une dizaine d'anueses, lorsqu'il dait a l'Institut Réovoth, avant de poursurve ses exsais anx IS.S.A.

On arrive maintenant is un prix de revient de $200 \mathrm{~F}$ au $\mathrm{m}^{*}$ d'eau par ce procédé, qui devient ainsi applicable a la production d'eau potable, mais non, bien entendu, a celle d'ean d'irrigation.

M. Marer observe qu'rsaiel est un des pays oi l'on vend le plus cher l'au d'irrigation: $30 \mathrm{f}$ le $\mathrm{m}^{3}$, mais un est lout de mime loin de $200 \mathrm{~F}$.

Ir. René Corss répond que da quantité d'eau mise, au moins theoriquement, a la disposition des Israeliens, est de 2000000000 de $\mathrm{m}^{3}$ par an, pour une population de 2000000 d'habitants. Il est curieux de rapprocher le cube moyen annuel par habitant, qui est donc de $1000 \mathrm{~m}$ die celui que l'on constate aux U.S.A., où, tous besoins confondus, y compris ceux de l'irrigation, on arrivait en 1955 a 180 milliards de $m^{3}$ par an. Il est vraisemblable gu'on atteint là un chiffre quj demeurera valable pendant un certain temps. Si les besoins s'aceroissent, dans tous les pays, en face de ressoures qui ne sont pas illimilees, on prend partout conscience du probleme de l'au et l'on s'oriente de plus en plus vers une politique d'economie. Israel peut done largement subsister avec ses ressources actuclles, mais se retrouvera devant le probleme de l'augmentation de ces ressources, lorsque sa population se sera a nouveau accrue dans une cerlaine proportion.

Nons ne sommes pas tres loin du moment où en muints endroits de la planete, les problèmes d'eau vont se poser sur le plan international. Ft nous n'y échapperons pas, en France même, pour nos régions de l'Est et du Nord. 